\title{
EDITORIAL
}

\section{On the Importance of Using a Multidimen- sional Sociotechnical Model to Study Health Information Technology}

\author{
Dean F. Sittig, $P b D^{1}$ \\ Joan $S$. Ash, $P b D^{2}$ \\ ${ }^{1}$ Memorial Hermann Center for Healthcare Quality \& Safety, National Center for Cognitive Informatics \& Decision Making, \\ School of Biomedical Informatics, University of Texas Health Sciences Center, Houston, Texas \\ ${ }^{2}$ Department of Medical Informatics \& Clinical Epidemiology, Oregon Health \& Science University, Portland, Oregon
}

Ann Fam Med 2011;9:390-391. doi:10.1370/afm.1291.

$\mathrm{T}$ The Crosson et $\mathrm{al}^{1}$ and Koopman et $\mathrm{al}^{2}$ articles in this issue provide excellent illustrations and validation of the need for a systematic approach to make health information technology (HIT) safe and effective for use within health care. These studies can be viewed in this comprehensive context through the lens of a recently developed 8 -dimension, sociotechnical model of HIT use. ${ }^{3,4}$ Briefly, this model offers a systems-level view and posits that all 8 of the interconnected dimensions of HIT must be adequately addressed if an organization is to achieve safe and effective electronic health record use. Failure to account for key items in any one of these dimensions can lead to almost insurmountable problems within the clinic.

The first aspect of this model, and the foundation of any e-prescribing or other HIT project, is represented by the hardware and software, which provide the features and functions the clinicians and office staff use to carry out the data entry, review, and transmission processes required to document their patient care activities. Crosson et al found in their observations that clinics required considerable on-site technical

Conflicts of interest: authors report none.

\section{CORRESPONDING AUTHOR}

Dean F. Sittig, PhD

School of Biomedical Informatics

University of Texas Health Sciences Center

6410 Fannin St, UTPB 1100.43

Houston, TX 77030-3006

dean.f.sittig@uth.tmc.edu resources to help them implement and maintain the required technical infrastructure.

The clinical content required to implement the medication lists, drug dictionaries, formulary and billing information, and medication history, along with the drug-drug interaction checks, represents the second dimension of the model. The Koopman et al article provides an excellent overview of the detailed clinical knowledge (ie, content) that must be collected, managed, and effectively brought to bear at the right point in the clinical workflow to help clinicians solve complex patient care problems. ${ }^{5}$

The human-computer user interface, the third dimension, is critical. The article by Koopman et al describes an effective, highly specialized, conditionspecific, human-computer interface developed to help clinicians in an ambulatory setting quickly and efficiently find all the information they need to provide high-quality diabetes care.

Certain special and essential personnel, represented by the fourth dimension, are required to design, develop or configure, implement, use, and evaluate all aspects of the HIT. The Koopman et al article highlights how clinically focused, human-computer interface evaluation experts can help clinicians evaluate new system functionality. The Crosson et al article illustrates the different skills and knowledge that are required to plan successfully (eg, clinical champions or opinion leaders), implement (eg, technical resources), and use (eg, superusers) such new HIT functions as e-prescribing. Often the incorporation of team members with complementary knowledge, skills, and experience can greatly improve the quality of the research 
and the implementation process and hence make the results or the system more useful. ${ }^{6}$

The fifth dimension of the model describes the communication and workflow processes into which any new or existing HIT functionality must fit. ${ }^{7}$ Crosson et al found that "successful adoption of e-prescribing required substantial investments of planning time and the ongoing transformation of work processes," along with the development of new communication strategies, to successfully implement e-prescribing. Likewise, and most impressively, Koopman et al's project developed a new user interface that reduced the mean number of mouse clicks required to complete the work task from 60 to 3 !

Internal policies, procedures, and culture of the organization represent the critical sixth dimension. The article by Crosson et al provides a view of the many organizational policies, procedures, and perhaps most challenging, the cultural changes that a practice must make to implement an e-prescribing system successfully. One of the most difficult changes involves the participation in newly created health information exchanges and interactions with external pharmacies. Both of these external communication pathways are required by the US federal government's new incentive programs. ${ }^{8}$ Such government programs include rules and regulations, the seventh dimension, which govern many of the system features, functions, and user actions. For example, the new user interface described by Koopman et al has the potential to reduce the cost and increase the quality and safety of the care provided. All of these benefits will be much more important should the currently dominant fee-for-service health care system be transformed into more of a payfor-performance environment. Likewise, the study by Crosson et al highlights the difficult challenges facing ambulatory practices interested in participating in the government's new meaningful use incentive plan.

The final dimension of the model covers all aspects of the measurement and monitoring required to identify problems, monitor system use and usability, and make the measurements required to evaluate the overall effect of the project. Both the Crosson et al and Koopman et al articles describe extensive system evaluations. The Crosson et al article reports on an external, multimethod, qualitative study of 5 ambulatory clinics using e-prescribing systems. The Koopman et al article describes use of a mobile usability labora- tory to assess the utility of a new user interface. The methods described in these 2 articles will be of interest to others trying to gain insight through careful evaluation of the development or implementation of new HIT functionality.

The point is that no matter how good your new HIT system in general or your clinical decision support interventions in particular are, if you do not have adequate hardware to run the application, access to the data, a good place in the workflow to inject the system, people to take the actions recommended by the computer, and a regulatory environment that will allow the implementation to proceed, you will have difficulties. Proactive planning and evaluation through this 8 -dimensional sociotechnical model of safe and effective HIT use can help organizations make sure that they have addressed all of the key requirements for any successful HIT project.

To read or post commentaries in response to this article, see it online at http://www.annfammed.org/cgi/content/full/9/5/390.

Key words: Medical informatics; electronic prescribing; user-computer interfaces; computer systems evaluation

Submitted June 2, 2011; submitted, revised June 2, 2011; accepted June 6, 2011.

\section{References}

1. Crosson JC, Etz RS, Wu S, Strauss SG, Eisenman D, Bell DS. Meaningful use of electronic prescribing in 5 exemplar primary care practices. Ann Fam Med. 2011;9(5):392-397.

2. Koopman RJ, Dochendorfer KM, Moore JL, et al. A diabetes dashboard and physician efficiency and accuracy in accessing data needed for high-quality diabetes care. Ann Fam Med. 2011;9(5)398-405.

3. Sittig DF, Singh $\mathrm{H}$. Eight rights of safe electronic health record use. JAMA. 2009;302(10):1111-1113.

4. Sittig DF, Singh $H$. A new sociotechnical model for studying health information technology in complex adaptive healthcare systems. Qual Saf Health Care. 2010;19(Suppl 3):i68-i74.

5. Sittig DF, Wright $A$, Simonaitis $L$, et al. The state of the art in clinical knowledge management: an inventory of tools and techniques. Int J Med Inform. 2010;79(1):44-57.

6. Ash JS, Stavri PZ, Dykstra R, Fournier L. Implementing computerized physician order entry: the importance of special people. Int J Med Inform. 2003;69(2-3):235-250.

7. Ammenwerth $\mathrm{E}$, Iller C, Mahler C. IT-adoption and the interaction of task, technology and individuals: a fit framework and a case study. BMC Med Inform Decis Mak. 2006;6:3.

8. Blumenthal D, Tavenner M. The "meaningful use" regulation for electronic health records. N Engl J Med. 2010;363(6):501-504. Epub2010Jul13. 